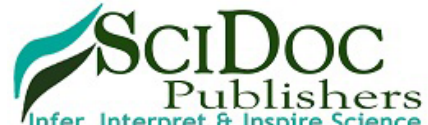

\author{
International Journal of Dentistry and Oral Science (IJDOS) \\ ISSN: 2377-8075
}

\title{
Effectiveness of a Novel Nano-Silver Fluoride with Green Tea Extract Compared with Silver Diamine Fluoride: A Randomized, Controlled, Non-Inferiority Trial
}

Research Article

Zuhair Al-Nerabieah ${ }^{1 *}$, Ettihad Abo Arrag ${ }^{2}$, John C Comisi ${ }^{3}$, Anas Rajab ${ }^{4}$

${ }^{1}$ Pediatric dentistry department, Dental Collage, Damascus University, Al-Mazzeh St. Damascus, Syria.

${ }^{2}$ Senior lecturer, Pediatric dentistry department, Dental Collage, Damascus University, Al-Mazzeh St. Damascus, Syria.

${ }^{3}$ Associate professor, Restorative Dentistry, Department of Oral Rehabilitation, Medical University of South Carolina, James B. Edwards College of Dental Medicine, Charleston, South Carolina, USA.

${ }^{4}$ Associate professor, Organic chemistry department, Faculty of Pharmacy, Syrian private University (SPU), Al-Mazzeh St. Damascus, Syria.

\section{Abstract}

The aim of this study wasto evaluate whether the cariostatic efficacy of a biologically synthesized novel Nano-Silver Fluoride with green tea extract (NSF-GTE) is non-inferior to Silver Diamine Fluoride (SDF) 38\% in deciduous teeth in preschool children. This study was a randomized, single-blinded, non-inferiority clinical trial. Sixty-three preschoolers with a total of 164 active lesions were selected and randomly assigned into two groups (A: 32 children with 83 lesions treated with NSF-GTE - B: 31 children with 81 lesions treated with SDF). Clinical evaluation was performed at 21 days, 3 and 6 months after treatment using International Caries Detection and Assessment System (ICDAS II) criteria to assess carious lesions activity. Non- Inferiority margin was set at $15 \%$. At six months, Total arrest rate was $67.4 \%$ and $79.6 \%$ for NSF-GTE and SDF respectively (P> 0.05). Furthermore, $95 \%$ confidence interval of the Relative Risk for (group A) at the three follow-up periods lies entirely below the predefined margin in comparison to (group B). Also, it was observed that anterior teeth and single surface lesions had higher arrest rates as compared to posterior teeth and multiple surface lesions $(\mathrm{P}<0.05)$. Non-Inferiority was demonstrated, and both SDF and NSF-GTE presented cariostatic efficacy in primary teeth.

Keywords: Nano-Silver; Fluoride; Green Tea; Silver Diamine Fluoride; Caries; Children.

\section{Introduction}

Despite the advanced milestones that have happened in preventive dentistry in the last two decades, tooth decay still a ubiquitous chronic disease around the world [1]. An enormous amount of dental caries lesions is left untreated since oral health care demands are beyond the capacities of the dental health care organizations [2]. In most deprived populations, this results in significant disease advancement that leads to ache, higher expenses, and decreased quality of life for the affected children and their families [3]. The development and implementation of cariostatic agents in deprived populations have been demanded by investigators and clinicians [4].

Recently, Silver Diamine Fluoride (SDF) has been demonstrated by various investigators, to be effective in arresting dentin carious lesions. However, the application of SDF creates dark black staining of caries tissues, which can be a significant drawback of its use, especially on anterior teeth [5].

Antimicrobial efficacy of Nano-Silver particles (AgNPs) against cariogenic bacteria have been demonstrated in-vitro studies [6]. Dos Santos et al. investigated the clinical efficacy of a chemically synthesized Nano Silver Fluoride (NSF). Even though it was effective in arresting dentin lesions without causing black stains [4], there is a concern regarding the hazardous chemical agents used as a reducing agent, and this has drawn attention to the safety of this product [7].

In contrast, Biological synthesis approach utilizing plant extracts have emerged as a simple alternative to typical chemical synthesis techniques [8]. The use of a green tea extract has recently become

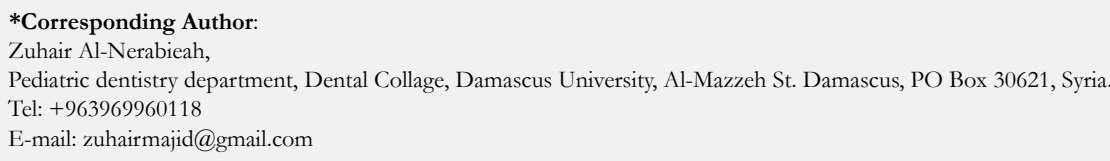


of great interest due to its favorable protocol, which is a fast, simple, eco-friendly, safe, and economically feasible green technique $[9,10]$. Green tea (Camellia Sinensis) is mainly consisted of polyphenolic compounds that act as a reducing and capping agent in the biological synthesis process of AgNPs [11].

To the extent of our knowledge, after reviewing the literature, there were no in-vivo studies that have evaluated the efficacy of biologically synthesized Nano-Silver Fluoride in arresting caries. Thus, this study aim was to investigate whether the cariostatic efficacy of a biologically synthesized novel Nano-Silver Fluoride with green tea extract (NSF-GTE) is non-inferior to Silver Diamine Fluoride (SDF) 38\% in primary teeth in preschool children.

\section{Materials and Methods}

Chemical substances were acquired from Sigma Aldrich (St. Louis, MO, USA), and green tea leaves purchased from a local market. Deionized water was prepared using a water deionizer machine (ZHUOYUE-1, Sichuan, China). Silver Diamine Fluoride 38\% was obtained from Elevate Oral care (Advantage Arrest ${ }^{\circledR}$, Elevate Oral Care LLC., West Palm Beach, FL, USA).

\section{Synthesis of Nano-Silver Fluoride with green tea extract (NSF-GTE)}

Preparation of green tea (Camellia sinensis) extract: "Green tea extract was prepared by exactly weighting $1.3 \mathrm{~g}$ of green tea leaves, which was transferred into a $250 \mathrm{ml}$ conical flask, already containing $100 \mathrm{~mL}$ of deionized water. The mixture was then heated at $80^{\circ} \mathrm{C}$ for $20 \mathrm{~min}$, and the extract was cooled at room temperature and then filtered by using Whatman ${ }^{\circledR}$ filter paper grade 1 (Healthcare GE, Boston, USA)". The filtered extract was used at the same session to synthesize Silver Nanoparticles [12].

Green Synthesis of Silver Nanoparticles (AgNPs): "60 $\mathrm{ml}$ of green tea extract was taken via pipette and added to $500 \mathrm{ml}$ of deionized water in a volumetric flask and hand-shaken to homogenize the dilution. The resulting $\mathrm{pH}$ of the solution was six and was further modified to a $\mathrm{pH}$ of 10 through the addition of potassium carbonate $\left(\mathrm{K}_{2} \mathrm{CO}_{3}\right)$. $20 \mathrm{ml}$ of the $1 \mathrm{mM} \mathrm{AgNO}$ aqueous solution was then added in a single shot." [12].
The formation of AgNPs was observed by the change of green tea extract color from yellow to dark brown after adding Silver Nitrate, which was reported in other studies $[8,9,12]$.

At the end of the biological synthesis of Ag-NPs, Sodium Fluoride $(\mathrm{NaF})$ was added to improve the stability and the cariostatic efficacy of the solution (10104 ppm) [4]. The final solution (NSFGTE) was stored in a dark amber bottle at $\left(4 \mathrm{C}^{\circ}\right)$ until further use.

Characterization of NSF-GTE: The morphology of NSFGTE was analyzed using Scanning Electron Microscopy (SEM) (VEGA II, TESCAN, CZE). SEM analysis showed that AgNPs were spherical with an average particle size of $4 \mathrm{~nm}$. (Figure 1)

\section{Clinical Trial}

This clinical study design was a randomized, single-blind, activecontrolled, parallel-group, non-inferiority trial. The study was conducted in accordance with the Declaration of Helsinki and ethical approval was obtained from the Institutional Review Board in Damascus University (R.N 2297). Also, the trial was registered in the Australian New Zealand Clinical Trial Registry (ANZCTR) (Trial Id: ACTRN12618001865202). The extension of the CONSORT statement for reporting non-inferiority trials was applied in this study [13].

\section{Study Setting}

The study was conducted between 2018 and 2019 in a single kindergarten in Damascus where the water supply has a very low concentration of Fluoride 0.03 ppm [14].

\section{Sample size}

The sample size was calculated with G*Power 3.1.9 software (University of Kiel, Germany). The significance level was set at 0.05 , the statistical power was set at $80 \%$ and the predefined noninferiority margin of carious lesions arrest was set at $15 \%$. Based on a student t-test and previous studies [4], an estimated 60 carious lesions in each group were required to demonstrate an effect size $(0.4)$ in the average proportion of arrested caries. The total sample size was raised by $20 \%$ to avert the adverse effect of the possible drop rate.

Figure 1. Scanning Electron Microscopy showing shape and size of the Silver nanoparticles synthesized with green tea extract.

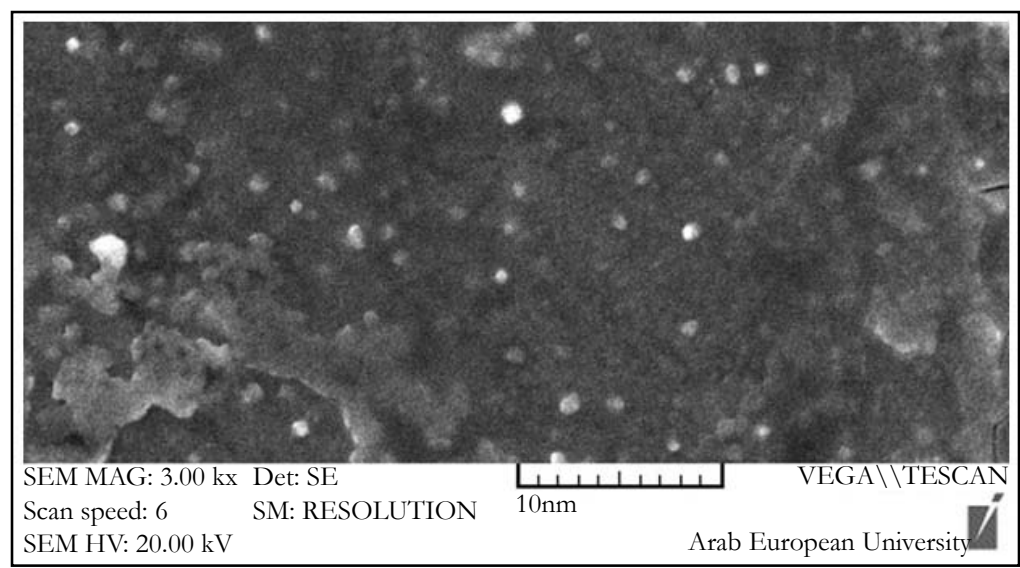




\section{Participants Recruitment}

A single Investigator screened 150 children for eligibility, depending on the following inclusion and exclusion criteria.

\section{Inclusion Criteria of participants:}

1. Healthy children willing to co-operate.

2. aged 36 to 60 months

3. Have not been treated with antibiotics in the past month before enrollment to avoid the possible hyposalivation effect [15].

\section{Inclusion Criteria of teeth:}

1. Each participant had at least one active lesion with dentin exposed based on the ICDAS II ("Code 5: dentin cavity easily visible with the naked eye where the surface of cavity feels soft or leathery on gentle probing") [16].

\section{Exclusion criteria:}

1. Children weighing less than $15 \mathrm{~kg}$ [17].

2. known sensitivity to Silver or other heavy-metal ions [18].

3. presence of gingivitis.

4. The tooth was excluded if it had pulp exposure, abscess/fistula, sensitivity to percussion or discoloration related to tooth nonvitality.
Written informed consent and an invitation letter that describes the scope and purpose of the research was sent to parents and guardians of eligible children. Children who were enrolled in this study had brought back a signed consent before the initiation of the trial.

Children in the kindergarten were supplied with written instructions for maintaining oral hygiene, toothbrush and a fluoridated toothpaste containing $600 \mathrm{ppm}$ of Fluoride.

\section{Randomized allocation}

Sixty-three children with a total of 164 active caries lesions met the inclusion criteria and were enrolled in this study. Each participant was assigned to one treatment group to avoid the possible cross-over effect of the NSF-GTE and SDF. Hence, children were allocated into two strata based on their carious lesion number (1-2 lesions or $\geq 3$ lesions).

Then, Statistical Package for the Social Sciences (SPSS) version 25.0 (IBM, Chicago, IL, USA) was utilized to generate a randomization schedule with stratification. After that, Children were randomly assigned to one of the groups with 1:1 ratio. This Randomized allocation process was performed by an external investigator to achieve allocation concealment. The Consort diagram that describes participant flow is outlined in Figure 2.

Figure 2. Consort flowchart showing number of teeth included in this study.

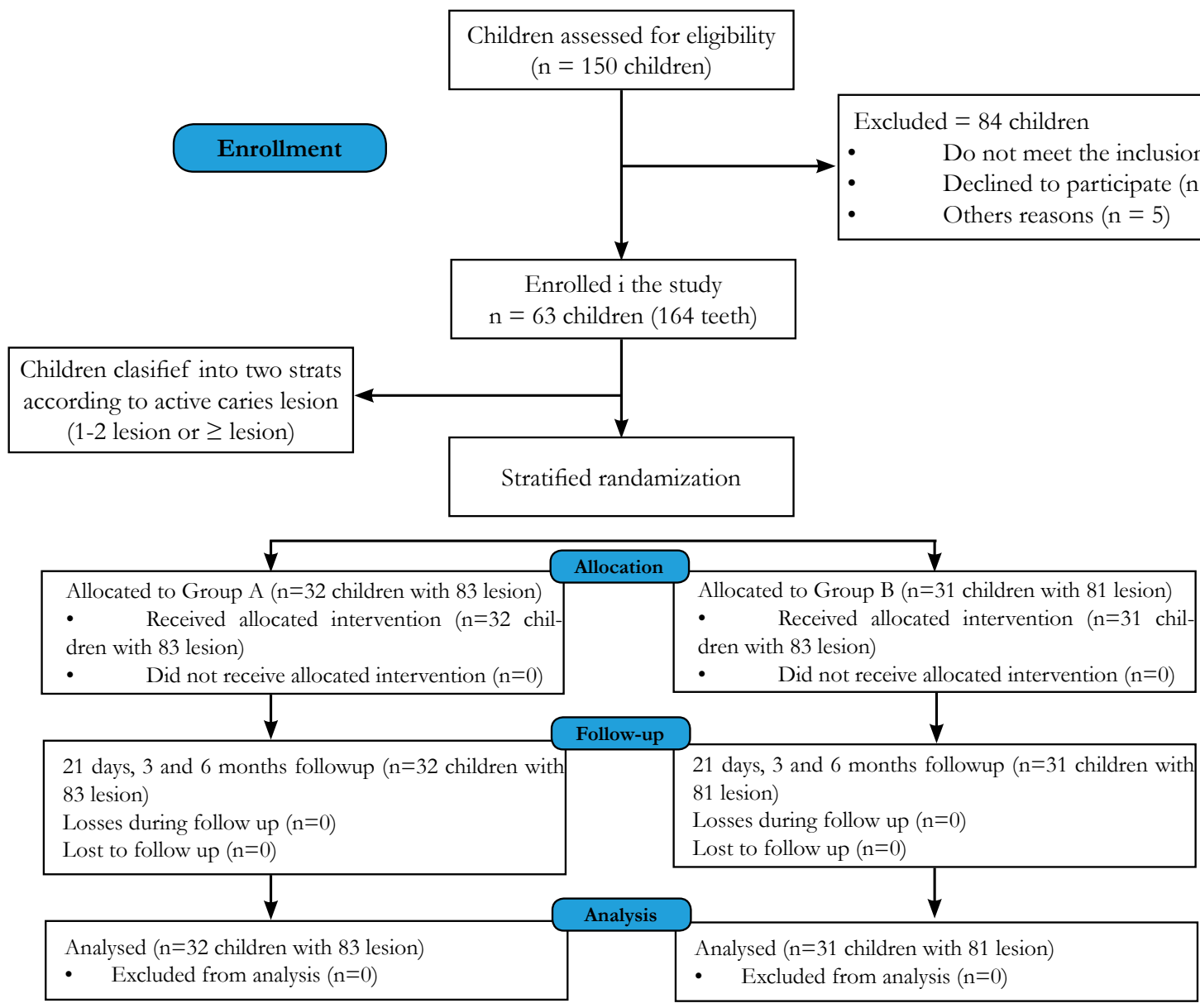




\section{Research Protocol}

Clinical examination was carried out by a single experienced investigator (AZ) using a disposable blunt probe and mirror. In the first session, decay, extracted due to caries, filled tooth (deft) index was scored for enrolled children to evaluate oral hygiene status. Then, caries activity assessment was carried out using visual and tactile inspection, according to ICDAS II. Teeth with easily detectable cavitated lesions, where the surface felt soft or leathery on gentle probing, were considered active cavitated lesion and enrolled in the study.

A total of 63 children with 164 lesions were stratified according to active carious lesions (1-2 lesions or $\geq 3$ lesions), then they were randomly allocated into one of the following groups:

- Group A: Nano-Silver Fluoride with green tea extract (NSFGTE)

- Group B: SDF

In the next visit, the affected tooth surfaces were gently cleaned by a disposable micro-brush applicator for at least 30 seconds and then dried with cotton gauze sponges. No effort was made to remove the carious tissue before the application of the agent. Gingival tissues of the targeted tooth were protected with petroleum jelly, and isolation was carried out using cotton rolls.

A new fine micro-brush (Micro brush international, Grafton, WI 53024, United States) was dipped into one of the agents (NSFGTE or SDF $)$ and $(0.1 \mathrm{ml})$ was applied to the lesion surface for one minute. The areas were not rinsed and the tooth surface was covered with petroleum jelly. Kindergarten teachers were instructed that participants were not allowed to eat or drink for an hour after the application (18). Examinations and intervention applications were carried out in one of the kindergarten classrooms and applied by the same investigator (AZ).

Clinical evaluation of the lesions was carried out using a visual and tactile assessment, which was performed 21 days, 3 and 6 months after treatment using (ICDAS II) criteria to assess carious lesions activity. The assessment was achieved by two blinded calibrated investigators using a blunt probe and artificial light. Shiny lesions that felt hard on gentle probing were considered arrested cavities according to ICDAS II criteria. $10 \%$ of the total sample was randomly re-evaluated on different occasions to monitor intra-examiner reproducibility.

\section{Blinding}

Participants, parents and biostatistician were blinded toward agents used in the study by using two identical bottles with tag codes A and B. Even though outcome examiners were not aware of which agent was used on the treated tooth, the blinding process was not guaranteed since the lesions treated with SDF were dark stained.

\section{Statistical analysis}

Since children with multiple carious lesions were enrolled, each tooth was treated as a single unit. The Kolmogorov-Smirnov test was used to analyze the normality distribution of quantitative data. Intragroup comparisons were made using the Chi-square test with a $95 \%$ confidence interval and a two-sample t-test was used for means comparisons.

For the non-inferiority of caries arrest, the null hypothesis was that (NSF-GTE) is inferior to the (SDF) by at least 15\%. Thus, the per-teeth percentage of active lesions at baseline treated with NSF-GTE versus SDF and stayed arrested throughout the 6-months follow-up was analyzed. The non-inferiority margin was set at $15 \%$ according to previous studies $[18,19]$. Confidence intervals were calculated for the difference between the two groups, with the width of this interval representing the margins of noninferiority. Even though this is the preferred method to report non-inferiority results according to the United States for Food and Drug Administration (FDA) and Consort statement [13, 20], But P-values were also reported for the rate of caries arrest between the two groups.

The Co-variables that could possibly alter the treatment effects on the arrest rate were assessed using a multi-level non-linear logistic regression model, which was built using Generalized Equation Estimation (GEE). The dependent variable was caries arrest, and Co-varieties were (Treatment group, age, gender, deft index, type of lesion, and site of the lesion). The level of significant difference was set at 0.05 for all statistical tests. Statistics were calculated using the (SPSS) software v25.0. Intra-rater reliability and inter-examiner agreement for caries assessment were calculated by Cohen's kappa test. The kappa for intra-rater reliability was 0.85 and 0.90 . The kappa for the inter-examiner agreement was 0.87 .

\section{Results}

Sixty-three preschool children (22 males and 39 females) were enrolled in this study with a mean age of 3.9 and 4.1 years for group $\mathrm{A}$ and $\mathrm{B}$ respectively. The mean (deft) index at baseline was (4.1 \pm 1.8) for group $A$ and $(5.1 \pm 0.5)$ for group $B$ with no statistically significant difference between the two groups $(\mathrm{P}>0.05)$. (Table 1) A total of 63 preschoolers with 164 cavitated active lesions were randomly allocated into two groups: 32 children with 83 lesions for the Nano-Silver Fluoride with green tea extract group (Group A) and 31 children with 81 lesions for the Silver Diamine Fluoride group (Group B).

The number, type, and site of lesions in each group were not identical because some participants had more than one lesion included in this trial, and the protocol required that every participant receive only one type of the agents to avoid the crossover effect. Nevertheless, there were no statistically significant differences between the groups ( $\mathrm{P}>0.05)$. (Table 1)

After 21 days, 77\% (95\% CI: 0.59 to 0.98 ) of active lesions in group A showed hard arrested dentin, compared with $90 \%$ (95\% CI: 0.706 to 1.13 ) of arrested lesions in the group $\mathrm{B}(\mathrm{P}>0.05)$. After three months, Group A and B had 71\% (95\% CI: 0.54 to 0.91$)$ and $85 \%(95 \% \mathrm{CI}: 0.66$ to 1.07$)$ arrest rate respectively $(\mathrm{P}>0.05)$. At 6 months, $67.4 \%$ (95\% CI: 0.50 to 0.87$)$ of active lesions treated with NSF-GTE were still arrested, while 79.6\% (95\% CI: 0.608 to 1.01$)$ active lesions treated with SDF group remained arrested (95\% CI: 0.608 to 1.01$)(\mathrm{P}>0.05)$. (Table 2$)$

Figure 3 demonstrates the non-inferiority of NSF-GTE as 
Table 1. Comparison of number and age of children enrolled, deft, type and site of active lesions.

\begin{tabular}{|c|c|c|c|}
\hline Variables & Group A $^{\mathbf{a}}$ & Group B $^{\mathbf{b}}$ & P value $^{*}$ \\
\hline Children enrolled & 32 & 31 & $0.73^{* *}$ \\
\hline Male & 12 & 17 & $0.83^{* *}$ \\
\hline Female & 20 & 14 & $0.90^{* *}$ \\
\hline Age (mean \pm SD) & $3.9 \pm 0.4$ & $4.1 \pm 0.5$ & $0.68^{* *}$ \\
\hline Deft (mean \pm SD) & $4.1 \pm 1.8$ & $5.1 \pm 0.5$ & $0.126^{* *}$ \\
\hline Active lesion & 83 & 81 & $0.797^{* *}$ \\
\hline Type of lesion & \multicolumn{3}{|l}{} \\
\hline Single surface $(\%)$ & $35(42 \%)$ & $32(40 \%)$ & \\
\hline Multiple surface $(\%)$ & $48(58 \%)$ & $49(60 \%)$ & $0.471^{* *}$ \\
\hline Site of lesion & \multicolumn{3}{|l}{} \\
\hline Anterior & $29(35 \%)$ & $28(34 \%)$ & \\
\hline Posterior & $54(65 \%)$ & $53(66 \%)$ & $0.256^{* *}$ \\
\hline
\end{tabular}

${ }^{a}$ : Nano-Silver Fluoride with green tea extract ${ }^{\text {b }}$ : Silver Diamine Fluoride *: two-sample t-test for means and chi-square for percentages ${ }^{* *}$ : Not significant

Table 2. Evaluation of Caries arrest rate at 21 days, 3 months and 6 months.

\begin{tabular}{|c|c|c|c|c|}
\hline Variables & Group Aa & Group Bb & ARR $^{\dagger}$ & P value* $^{*}$ \\
\hline 21 days & \multicolumn{5}{|l|}{} \\
\hline Arrested & $64(77 \%)$ & $73(90 \%)$ & & \\
\hline Active & $19(23 \%)$ & $8(10 \%)$ & 0.13 & $\mathrm{P}=0.361^{* *}$ \\
\hline $95 \% \mathrm{CI}^{\ddagger}$ & 0.593 to 0.984 & 0.706 to 1.13 & & \\
\hline $\mathbf{3}$ months & $59(71 \%)$ & $69(85 \%)$ & & \\
\hline Arrested & $24(29 \%)$ & $12(15 \%)$ & 0.14 & $\mathrm{P}=0.306^{* *}$ \\
\hline Active & \multicolumn{5}{|l|}{} & \\
\hline $95 \%$ CI & 0.541 to 0.916 & 0.662 to 1.07 & & \\
\hline $\mathbf{6}$ months & \multicolumn{5}{|l|}{} \\
\hline Arrested & $56(67.4 \%)$ & $64(79 \%)$ & & \\
\hline Active & $27(32.6 \%)$ & $17(21 \%)$ & 0.11 & $\mathrm{P}=0.387^{* *}$ \\
\hline $95 \% \mathrm{CI}^{\ddagger}$ & 0.509 to 0.876 & 0.608 to 1.01 & & \\
\hline
\end{tabular}

a: Nano-Silver Fluoride with green tea extract b: Silver Diamine Fluoride

t: Absolute Risk Reduction

¥: 95\% confidence interval for average proportion of arrested caries and difference in average proportion of arrested caries. ${ }^{*}$ : chi-square ${ }^{* *}$ : Not significant

Figure 3. Non-Inferiority plot showing $95 \%$ CI difference between NSF-GTE and SDF at three follow-up periods.

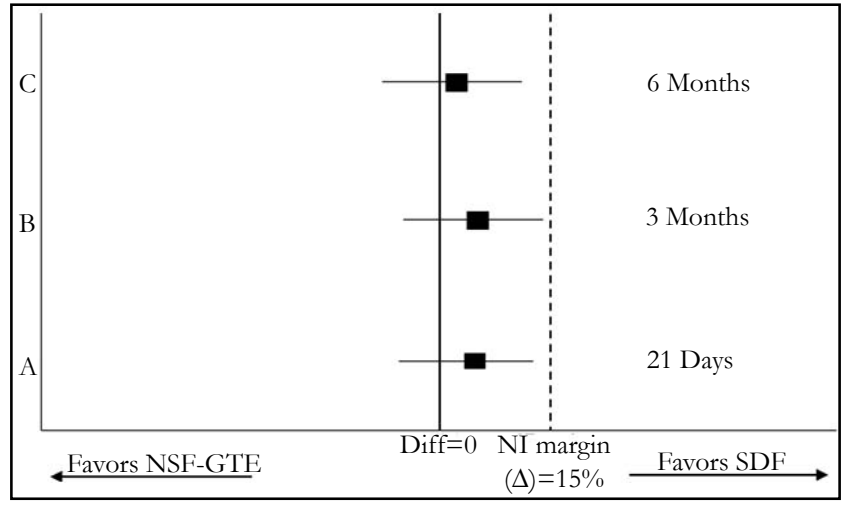


it shows that $95 \%$ confidence interval of the Relative Risk for (group A) at the three follow-up periods lies entirely below the predefined margin in comparison to (group B).

In the multi-level non-linear logistic regression model, results of age, gender, deft index, and treatment group were not significant. However, the type of lesion and the site of the lesion have affected the caries arrest rate significantly. Regarding the type and size of the lesions, single surface lesions had 1.99 times the chance to become arrested compared to multiple-surface lesions $(\mathrm{P}<$ 0.05). Whereas lesions located in anterior teeth had 3.15 times the chance to become arrested compared to posterior lesions $(\mathrm{P}<$ 0.05). (Table 3)

After six months, all lesions treated by SDF (group B) have turned black (Figure. 4a). While lesions of the NSF-GTE (group A) have not in any way turned black. (Figure. 4b)

Table 3. Multi-level Logistic regression model of the caries arrest rate after 6 months with clustering effect.

\begin{tabular}{|c|c|c|c|}
\hline Variables & Odds Ratios $^{\dagger}$ & $\mathbf{9 5 \%}$ CI & P value \\
\hline Type of lesion & \multicolumn{3}{|l|}{} \\
\hline Single surface vs Multiple surfaces & & & \\
\hline Site of lesion & 1.99 & 0.917 to 4.34 & $0.029^{*}$ \\
\hline Anterior vs posterior $^{\ddagger}$ & 3.15 & 1.85 to 5.79 & $0.003^{*}$ \\
\hline
\end{tabular}

t: An odds ratio of more than 1 means that there is a higher arrest chance in the test group while an odds ratio of lower than 1 means that there is a lower arrest chance in the test group.

‡: Reference point*: Statistically significant

Figure 4. a) Arrested cavitated lesions treated with NSF-GTE after 6 months b) Arrested cavitated lesions treated with SDF after 6 months.

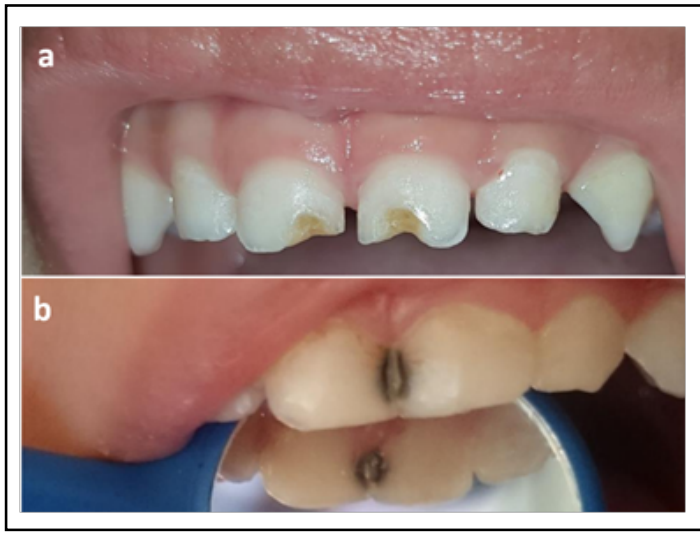

\section{Discussion}

Young children, in impoverished countries with limited access to health care, suffer from a high incidence of having untreated carious lesions [21]. Thus, the age group that was chosen to be studied in this trial was between 3 and 5 years old.

A biological approach using Green tea extract was used in this study to synthesize AgNps instead of the chemical approach that was reported by other studies [4, 22]. This new eco-friendly "green" method of synthesis offers a simple, fast, and a one-step (biogenic) approach. Studies have shown that green tea extract can act as a reducing and capping agent for the production of AgNPs without the need for adding hazardous chemicals or complicated laboratory devices $[8,9,23]$. On the other hand, chemical synthesis of nano-Silver particles has raised questionable arguments since the process contains hazardous substances such as acetic acid and borohydride sodium [7].

Also, SDF was chosen as an active control since this topical agent is recently considered the gold standard in arresting dentin caries in primary teeth [5].
This controlled trial was designed as a non-inferiority trial to test whether the effect of (NSF-GTE) is not exorbitantly worse than the effect of SDF $38 \%$ by more than a predefined non-inferiority margin $(15 \%)$. This design is crucial when the test drug cannot be compared to a placebo for ethical reasons, especially if this indicate negating participants an effective drug treatment. Furthermore, "non-inferiority is used when the test drug is believed to have slightly worse efficacy compared to the active control but offers safer, more cost-effective, or better esthetics options treatment options"[20].

International Caries Detection and Assessment System (ICDAS II) criteria have been applied in the inclusion and the outcome assessment in this study. Cavities that had changed from soft or leathery into shiny and hard were considered arrested caries. These criteria have been widely studied, and it was shown that ICDAS II criteria are reliable, valid, and reproducible in assessing the activity of primary caries [24-28].

In this trial, the first period of follow-up at 21 days after agents' application is used, to be in accordance with the reported period 
(21 days) required for Silver Diamine Fluoride to arrest caries [29, 30]. On the other hand, there are no studies about the efficacy of NSF-GTE nor the time it needs to arrest caries. Therefore 3 and 6 months follow up periods were included to identify the effect of this agent after more extended periods post-application.

The arrest rate for carious lesion treated with SDF was noticeably higher than NSF-GTE in all follow-up periods. This increased rate is likely attributable to the fact that SDF 38\% contains much higher concentrations of Silver Nitrate and Sodium Fluoride than those in NSF-GTE (5x AgNO3 \%, 4x NaF \%) [31]. However, the difference in the arrest rate was not statistically significant, and this trial demonstrated the non-inferiority of NSF-GTE compared to SDF.

This trial is the first "in-vivo study" to assess the effectiveness of a novel Nano-Silver Fluoride with green tea extract (NSF-GTE) to arrest cavitated lesions. The present study shows that NSFGTE was effective in arresting dentin carious lesions in both anterior and posterior primary teeth with the total arrest rate after six months $(67.4 \%)$.

A valuable component of green tea is the polyphenols. Polyphenols possess an antibacterial property that is attributable to the active ingredient, Catechins. The anti-cariogenic effect of green tea extract includes many activities [32]. Green tea can prevent the adhesion of bacteria on the tooth surface [33]. Furthermore, green tea catechins own a suppressing effect on the development of S. mutans, which in turn can decrease glucosyltransferase activity and reduce capsule synthesis $[34,35]$. Also, catechins can reduce plaque acidity and maintain $\mathrm{pH}$ towards neutrality, which is an unfavorable environment for cariogenic bacteria [36].

Silver Nanoparticles (AgNPs) are being widely used for its broadspectrum antibacterial property. Two approaches can explain the possible mechanisms of action of Nano-Silver particles. The first one is the interaction of the AgNPs with the bacterial cell membrane. AgNPs can establish pits in the cytoplasmic membrane of the bacteria through reactive oxygen species (ROS), which can lead to cell death [37].

Second, AgNPs penetrate the membrane followed by a burst release of Silver ions inside the bacterial cells, which "interacts with the DNA replications, cell inclusions, enzymes and disrupts the respiratory chain reactions causing inhibition of cell division, which may ultimately cause cell death"[38].

In vitro microbial studies showed that AgNPs were effective in inhibiting S. mutans strains, one of the primary pathogens responsible for dental caries. AgNPs are both bacteriostatic and bactericidal $[39,40]$. Furthermore, it has been reported that the antibacterial efficacy of AgNPs increases with smaller particle size and spherical shape by providing a larger surface area for contact with bacteria [41]. SEM analysis of the AgNPs in this study showed spherical AgNPs with approximate sizes of $8 \pm 4.4 \mathrm{~nm}$.

To the best of our knowledge, there are only two in vivo studies in the literature reporting the efficacy of Chemically synthesized Nano-Silver Fluoride in arresting dentin carious lesions in primary teeth. Dos Santos et al. reported an arrest rate of $66.7 \%$ by using chemically synthesized Nano Silver Fluoride preparation, which is equivalent to results obtained in this study $(67.4 \%$ arrest rate) [4]. To the contrary, Tirupathi et al. showed a higher arrest rate in Nano-Silver incorporated Sodium Fluoride (NSSF) group (77\%) [22]. This increased rate of arrested lesions could be attributed to two factors. First, the participating children in Tirupathi trial were older living in an optimal fluoridate community. Second factor, Silver nitrate concentration was higher $(5 \%)$ which could have played huge role in the arrest rate difference.

The Silver Diamine Fluoride arrest rate in this study $(79.6 \%)$, is consistent with other trials reports $[18,22]$. However, other studies have reported an average arrest rate as low as $43 \%$ after six months of treating carious lesions with SDF $[19,42]$. This could be attributed to our study application protocol, which consisted of applying topical agents for at least one minute and then sealing cavity with petroleum gel so the agent could stay in contact with the lesion as long as possible. While in Zhi and Duangthip trials, SDF was applied for only 10s, and then it was washed away from the cavity $[19,42]$.

In this study, primary anterior and posterior teeth with single or multiple surface lesions were included. It was observed that anterior teeth and single surface lesions showed higher arrest rates in comparison to posterior teeth and multiple surface lesions. This result was statistically significant and can be justified by the fact that molars and multiple surface lesions have a higher propensity for plaque accumulation and food impaction. Even more, this result stems from the fact that incisors and single surface lesions are easier to clean by the parents for this age group.

In vitro studies have shown the rationality that stands behind the safety of using NSF-GTE in this study. The use of green tea polyphenols to synthesize AgNPs is accountable for reducing AgNPs that serve as an effective antioxidant and anti-inflammatory agents, reducing the possible toxicity of the nanoparticles [8].

In the same context, in-vitro study showed that Silver nanoparticles synthesized from green tea extract at concentrations up to 100 $\mu \mathrm{g} / \mathrm{mL}$ did not exhibit cytotoxicity to $\mathrm{HaCaT}$ cells and did not induce changes in cellular morphology [43]. Another study reported higher IC50 values for biosynthesized AgNPs against normal human dermal fibroblasts, which indicates their lower toxicity in normal cell lines [44]. Targino et al., evaluated the cytotoxicity of different concentrations of Nano-Silver solutions that ranged from $(1 \%$ to $5 \%)$, and they found no toxic effect for erythrocyte [39].

The limitations of this study included a relatively short follow-up period of six months for the assessment of caries arrest. additionally, the investigator could not be blinded during the application process as a result of the different odor and color of both materials. Furthermore, confounding variables like dietary control and oral hygiene could not be monitored in the participating children. It is worth noting that after finalizing the trial, patients with the remaining active carious lesion in both groups were referred for treatment in the pediatric dentistry department.

\section{Conclusions}

Taken together, this trial demonstrated that Nano-Silver Fluoride with green tea extract (NSF-GTE) was non-inferior to Silver Diamine Fluoride (SDF) in arresting cavitated active carious le- 
sions in primary teeth in preschool children in a non-fluoridated community. NSF-GTE can be considered as an option to treat decayed primary teeth in underprivileged communities since it is simple, cost-effective for mass production, and does not necessitate sensitive application technique.

Further studies with different concentrations and different interval application with a larger sample size are required to assess the best protocol for Nano-Silver Fluoride with green tea extract application are needed.

\section{Author Contributions}

Zuhair Al-Nerabieah Contributed to conception, design, data acquisition, and interpretation, drafted and critically revised the manuscript; Ettihad Abo Arrag conception, design, data interpretation, drafted and critically revised the manuscript; Anas Rajab contributed to design, *Title Page with Author Details data interpretation and critically revised the manuscript; John C Comisi contributed, edited and critically revised the manuscript All authors have read and agreed to the published version of the manuscript."

\section{Funding}

“This research was funded by Damascus University).

\section{Acknowledgments}

The authors are grateful to the faculty of pharmacy at Syrian Private University (SPU) for the experimental support. Special thanks for Muaz AlKhouli and Salma Al Nesser for serving as examiners in the trial. We thank Safaa Shehabi for her assistance with the SEM measurements.

\section{References}

[1]. Vos T, Abajobir AA, Abate KH, Abbafati C, Abbas KM, Abd-Allah F, et al. Global, regional, and national incidence, prevalence, and years lived with disability for 328 diseases and injuries for 195 countries, 1990-2016: a systematic analysis for the Global Burden of Disease Study 2016. Lancet. 2017:390:1211-59. PMID: 28919117.

[2]. Organisation for Economic Co-operation and Development. Health at a Glance 2011: OECD Indicators. OECD/Korea Policy Centre, Korea; 2012.

[3]. Chaffee BW, Rodrigues PH, Kramer PF, Vítolo MR, Feldens CA. Oral health-related quality-of-life scores differ by socioeconomic status and caries experience. Community Dent Oral Epidemiol 2017;45:216-24.

[4]. Dos Santos VE, Filho AV, Ribeiro Targino AG, Pelagio Flores MA, Galembeck A, Caldas AF, et al. A new "silver-Bullet" to treat caries in children Nano Silver Fluoride: A randomised clinical trial. J Dent 2014;42:945-51.

[5]. Crystal YO, Niederman R. Evidence-Based Dentistry Update on Silver Diamine Fluoride. Dent Clin North Am 2019;63: 45-68. PMID: 30447792.

[6]. Hernández-Sierra JF, Ruiz F, Cruz Pena DC, Martínez-Gutiérrez F, Martínez AE, de Jesús Pozos Guillén A, et al. The antimicrobial sensitivity of Streptococcus mutans to nanoparticles of silver, zinc oxide, and gold. Nanomedicine Nanotechnology. 2008;4: 237-40. PMID: 18565800.

[7]. Burns J, Hollands K. Nano Silver Fluoride for preventing caries. Evid Based Dent. 2015;16:8-9. PMID: 25909929.

[8]. Rolim WR, Pelegrino MT, de Araújo Lima B, Ferraz LS, Costa FN, Bernardes JS, et al. Green tea extract mediated biogenic synthesis of silver nanoparticles: Characterization, cytotoxicity evaluation and antibacterial activity. Appl Surf Sci. 2019;463:66-74.

[9]. Kumar V, Wadhwa R, Kumar N, Maurya PK. A comparative study of chemically synthesized and Camellia sinensis leaf extract-mediated silver nanoparticles. 3 Biotech. 2019;9:7.

[10]. Ruttkay-Nedecky B, Skalickova S, Kepinska M, Cihalova K, Docekalova M, Stankova M, et al. Development of New Silver Nanoparticles Suit- able for Materials with Antimicrobial Properties. J Nanosci Nanotechnol 2019;19:2762-9.

[11]. Lorenzo JM, Munekata PES. Phenolic compounds of green tea: Health benefits and technological application in food. Asian Pac J Trop Biomed. 2016;6:709-19.

[12]. Elbossaty WF. Green Tea as Biological System for the Synthesis of Silver Nanoparticles. J Biotechnol Biomater 2017;7:2.

[13]. Piaggio G, Elbourne DR, Pocock SJ, Evans SJW, Altman DG, CONSORT Group for the. Reporting of noninferiority and equivalence randomized trials: extension of the CONSORT 2010 statement. Jama. 2012;308:2594604.

[14]. Beiruti N, Taifour M. Prevalence of nursing caries among children 3-5 years old in Damascus. East Mediterr Heal J. 2000;6:500-6. PMID: 11556044.

[15]. Bakhtiari S, Sehatpour M, Mortazavi H, Bakhshi M. Orofacial manifestations of adverse drug reactions: A review study. Clujul Med. 2018;91:27. PMID: 29440948.

[16]. Gugnani N, Pandit IK, Srivastava N, Gupta M, Sharma M. International caries detection and assessment system (ICDAS): A new concept. Int J Clin Pediatr Dent. 2011;4:93. PMID: 27672245.

[17]. ATSDR A for TS and DR. Toxicological profile for silver. Toxicol Profile Silver, US Dep Heal Hum Serv Public Heal Serv Atlanta, GA, 1990. (accessed July 27, 2019).

[18]. Milgrom P, Horst JA, Ludwig S, Rothen M, Chaffee BW, Lyalina S, et al. Topical silver diamine fluoride for dental caries arrest in preschool children: A randomized controlled trial and microbiological analysis of caries associated microbes and resistance gene expression. J Dent. 2018;68:72-8. PMID: 28866468.

[19]. Zhi QH, Lo ECM, Lin HC. Randomized clinical trial on effectiveness of silver diamine fluoride and glass ionomer in arresting dentine caries in preschool children. J Dent. 2012;40:962-7. PMID: 22892463.

[20]. Althunian TA, de Boer A, Groenwold RHH, Klungel OH. Defining the noninferiority margin and analysing noninferiority: An overview. $\mathrm{Br} \mathrm{J}$ Clin Pharmacol. 2017;83:1636-42. PMID: 28252213.

[21]. Kassebaum NJ, Bernabé E, Dahiya M, Bhandari B, Murray CJL, Marcenes W. Global burden of untreated caries: a systematic review and metaregression. J Dent Res. 2015;94:650-8.PMID: 25740856.

[22]. Tirupathi S, Nirmala SVSG, Rajasekhar S, Nuvvula S. Comparative cariostatic efficacy of a novel Nano-silver fluoride varnish with $38 \%$ silver diamine fluoride varnish a double-blind randomized clinical trial. J Clin Exp Dent. 2019;11: e105-12. PMID: 30805113.

[23]. Loo YY, Chieng BW, Nishibuchi M, Radu S. Synthesis of silver nanoparticles by using tea leaf extract from Camellia sinensis. Int J Nanomedicine. 2012;7:4263-7. PMID: 22904632.

[24]. Shoaib L, Deery CM, Ricketts DNJ, Nugent ZJ. Validity and reproducibility of ICDAS II in primary teeth. Caries Res. 2009;43:442-8.

[25]. Braga MM, Ekstrand KR, Martignon S, Imparato JCP, Ricketts DNJ, Mendes FM. Clinical performance of two visual scoring systems in detecting and assessing activity status of occlusal caries in primary teeth. Caries Res. 2010;44:300-8. PMID: 20530964.

[26]. Ekstrand KR, Luna LE, Promisiero L, Cortes A, Cuevas S, Reyes JF, et al. The reliability and accuracy of two methods for proximal caries detection and depth on directly visible proximal surfaces: an in vitro study. Caries Res. 2011;45:93-9. PMID: 21412000.

[27]. Tikhonova SM, Feine JS, Pustavoitava NN, Allison PJ. Reproducibility and diagnostic outcomes of two visual-tactile criteria used by dentists to assess caries lesion activity: a cross-over study. Caries Res. 2014;48:126-36. PMID: 24335157.

[28]. Dikmen B. Icdas II criteria (international caries detection and assessment system). J Istanbul Univ Fac Dent. 2015;49:63-72. PMID: 28955548.

[29]. Liu BY, Lo ECM, Li CMT. Effect of silver and fluoride ions on enamel demineralization: a quantitative study using micro-computed tomography. Aust Dent J. 2012;57:65-70. PMID: 22369560.

[30]. Zhi QH, Lo ECM, Kwok ACY. An in vitro study of silver and fluoride ions on remineralization of demineralized enamel and dentine. Aust Dent J. 2013;58:50-6. PMID: 23441792.

[31]. Crystal YO, Niederman R. Silver diamine fluoride treatment considerations in children's caries management. Pediatr Dent. 2016;38:466-71. PMID: 28281949.

[32]. Hamilton-Miller JMT. Anti-cariogenic properties of tea (Camellia sinensis). J Med Microbiol. 2001;50:299-302.

[33]. Ferrazzano GF, Roberto L, Amato I, Cantile T, Sangianantoni G, Ingenito A. Antimicrobial properties of green tea extract against cariogenic microflora: an in vivo study. J Med Food. 2011;14:907-11.

[34]. Subramaniam P, Eswara U, Reddy KRM. Effect of different types of tea on Streptococcus mutans: An in vitro study. Indian J Dent Res 2012;23:43.

[35]. Tao D-Y, Shu C-B, Lo ECM, Lu H-X, Feng X-P. A randomized trial on the inhibitory effect of chewing gum containing tea polyphenol on caries. J Clin 
Pediatr Dent. 2013;38:67-70.

[36]. Ahmed SI, Sudhir KM, Reddy VCS, Kumar RVSK, Srinivasulu G. Green Tea in the Prevention of Dental Caries A Systematic Review. Int Arch Biomed Clin Res 2017;3:1-6.

[37]. Morones JR, Elechiguerra JL, Camacho A, Holt K, Kouri JB, Ramírez JT, et al. The bactericidal effect of silver nanoparticles. Nanotechnology. 2005;16:2346-53. PMID: 20818017.

[38]. Arya G, Sharma N, Mankamna R, Nimesh S. Antimicrobial Silver Nanoparticles: Future of Nanomaterials. Microb. Nanobionics, Springer. 2019; 89-119.

[39]. Targino AGR, Flores MAP, dos Santos Junior VE, Bezerra F de GB, de Luna Freire H, Galembeck A, et al. An innovative approach to treating dental decay in children. A new anti-caries agent. J Mater Sci Mater Med. 2014;25:2041-7. PMID: 24818873.

[40]. Hernández-Sierra JF, Salas-López EK, Martínez-Gutiérrez F, Ruíz F, Pierdant-Pérez M, Mandeville P, et al., Bactericidal capacity of silver nanoparticles associated with Gantrez S-97 on Streptococcus mutans. J Clin Pedi- atr Dent. 2010;35:183-5. PMID: 21417121.

[41]. Agnihotri S, Mukherji S, Mukherji S. Size-controlled silver nanoparticles synthesized over the range 5-100 nm using the same protocol and their antibacterial efficacy. RSC Adv. 2014;4:3974-83.

[42]. Duangthip D, Chu CH, Lo ECM. A randomized clinical trial on arresting dentine caries in preschool children by topical fluorides-18 month results. J Dent. 2016;44:57-63. PMID: 26037274.

[43]. Moulton MC, Braydich-Stolle LK, Nadagouda MN, Kunzelman S, Hussain SM, Varma RS. Synthesis, characterization and biocompatibility of "green" synthesized silver nanoparticles using tea polyphenols. Nanoscale. 2010;2:763-70. PMID: 20648322.

[44]. Selvan DA, Mahendiran D, Kumar RS, Rahiman AK. Garlic, green tea and turmeric extracts-mediated green synthesis of silver nanoparticles: Phytochemical, antioxidant and in vitro cytotoxicity studies. J Photochem Photobiol B Biol. 2018;180:243-52. 